\title{
Environmental toxicology and biogeochemistry of ecosystems
}

\section{Muhammad Aqeel Ashraf $^{1,2} \cdot$ Muhammad Faheem $^{1}$}

Published online: 12 April 2020

(C) Springer-Verlag GmbH Germany, part of Springer Nature 2020

The fresh environment has comprised of clean air, soil, and water elements without human intervention or pollution involvement. The mixing of unwanted pollutants into these elements leads to the contamination of a fresh environment and disturbance of the ecosystem (Ashraf and Hanfiah 2017). There might be several routes including natural and anthropogenic activities that contributed to environmental deterioration. These routes include volcanic eruption, mineral weathering, fossil fuel burning, forests destroying, and industrial and agricultural activities. However, human intervention has reflected more provoking deleterious impacts on environmental quality and ecosystem sustainability (Häder et al. 2020). Pollutants such as trace elements (TEs), heavy metals (HMs), VOCs, $\mathrm{PM}_{1-10}$, microplastic, crude oil, nutrients, and synthetic organic materials (chemicals) especially emerging contaminants (ECs) including endocrine-disrupting compounds (EDCs) have been considered the main threat to the ecosystem due to their toxicological effects in all animal species to date (Gioia et al. 2011). Besides, environmental toxicology promotes wildlife mortality, which results in biodiversity issues. All around the globe, the contaminated sites proliferate everywhere. Therefore, there is a need to assess the technical feasibility of suggested solutions for the sake of maintaining environmental quality and ecosystem health.

Due to the persistent nature of pollutants, some of them like TEs, HMs, PCBs, and PAHs can accumulate in the environment and finally become a part of the food chain through feed crops intake, which results in health risks like respiratory,

Responsible Editor: Philippe Garrigues

Muhammad Aqeel Ashraf

chemaqeel@gmail.com

1 Department of Environmental Science and Engineering, School of Environmental Studies, China University of Geosciences, Wuhan 430074, China

2 International Water, Air \& Soil Conservation Society, 59200 Kuala Lumpur, Malaysia cardiovascular, and skin issues as well as neurotoxicity effect especially in human beings (Rhind 2009). For example, TEs have a tremendous role in the biogeochemical cycle. The TEs are available in plenty in various ecosystems both through natural and anthropogenic means. A detailed understanding of the release of TEs both under dynamic aerobic and anaerobic conditions prevail in deltaic soils is the key parameter to focus, as their increased movement through various mobilization routes is responsible for the availability of their higher concentrations in food crops (Rinklebe et al. 2017). The intake of these contaminated crops as a diet source becomes a cause of health risks to human beings, although the effect of individual chemicals on its exposure could disrupt normal functions (Fig. 1). However, the exposure of a combination of a variety of pollutants at once can be more effective than individual chemicals even at very low concentrations (Binetti et al. 2008). The extent of the biological effects of pollutant exposure relies on the pattern of exposure, way of uptake, and sensitivity of exposed target organs. At present, little information exists about the mobilization and biodegradation of pollutants as well as their fate and transport in the atmosphere, which need further detail investigation.

Environmental toxicology results in health risk issues and environmental deterioration around the globe as well as ecosystem imbalance, which becomes a cause of global pollution that is considered relatively new and a genuine worrying aspect. Environmental degradation is becoming a serious threat for mankind and living organisms due to rapid changes in industrialization and urbanization to cope with the needs of people in all developing countries around the globe. Therefore, the protection of our environment and conservation of the ecosystem are vital aspects that should be considered for the sake of sustainable development (Zhang and $\mathrm{Xu}$ 2011). Attempts of proper investigative approaches should be taken in the direction of understanding the possible acute toxic effects of such chemicals because of environmental toxicology on individuals as well as on ecosystems. Diverse experimental approaches should 


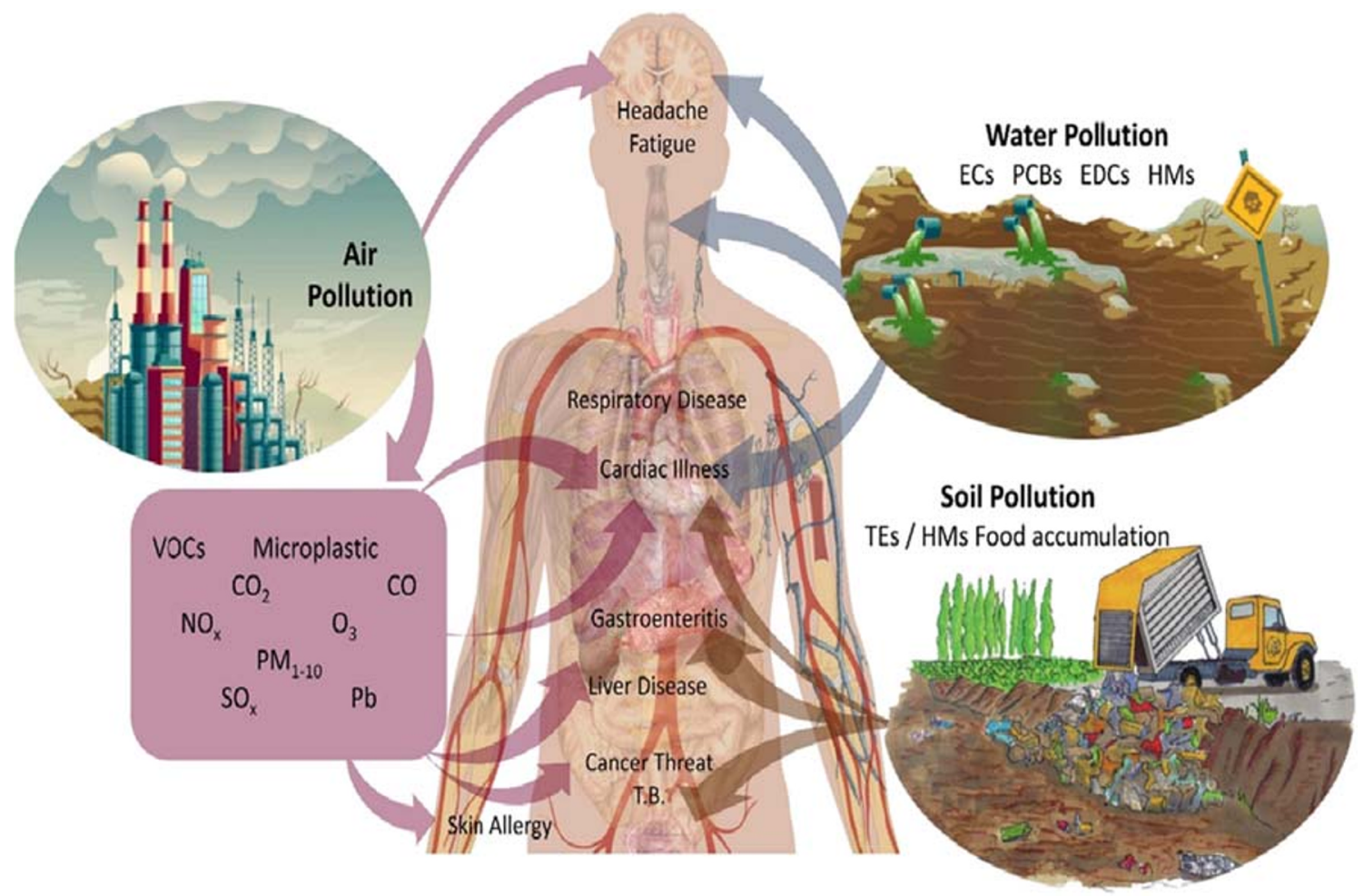

Fig. 1 Pollutants in the ecosystem

be adopted for the overall understanding of the ecosystem because individual investigations only focus on specific questions and it did not address all questions through different angles (Watson et al. 2016).

These entire dilemmas required very stringent policies and legislation from policymakers to maintain the wastewater stream's quality, clean atmospheric environment, and pure soil medium (Lafferty and Hovden 2003). The launched policies and regulations must assure the source control, implement the most recently available research outcomes, and follow successful feasible mitigation measures for the sake of remediation purposes.

\section{References}

Ashraf MA, Hanfiah MM (2017) Recent advances in assessment on clear water, soil and air. Springer

Binetti R, Costamagna FM, Marcello I (2008) Exponential growth of new chemicals and evolution of information relevant to risk control. Annali-Istituto Superiore di Sanità 44:13
Gioia R et al (2011) Sources, transport and fate of organic pollutants in the oceanic environment. In: Persistent Pollution-Past. Springer, Present and Future, pp 111-139

Häder D-P, Banaszak AT, Villafañe VE, Narvarte MA, González RA, Helbling EW (2020) Anthropogenic pollution of aquatic ecosystems: emerging problems with global implications. Science of the Total environment: 136586

Lafferty W, Hovden E (2003) Environmental policy integration: towards an analytical framework. Env Polit 12:1-22

Rhind S (2009) Anthropogenic pollutants: a threat to ecosystem sustainability? Philosophical Transactions of the Royal Society B: Biological Sciences 364:3391-3401

Rinklebe J, Kumpiene J, Du Laing G, Ok Y-S (2017) Biogeochemistry of trace elements in the environment-editorial to the special issue. $\mathrm{J}$ Environ Manag 2:127-130

Watson SC, Paterson DM, Queirós AM, Rees AP, Stephens N, Widdicombe S, Beaumont NJ (2016) A conceptual framework for assessing the ecosystem service of waste remediation: in the marine environment. Ecosystem services 20:69-81

Zhang M, Xu J (2011) Nonpoint source pollution, environmental quality, and ecosystem health in China: introduction to the special section. J Environ Qual 40:1685-1694

Publisher's note Springer Nature remains neutral with regard to jurisdictional claims in published maps and institutional affiliations. 


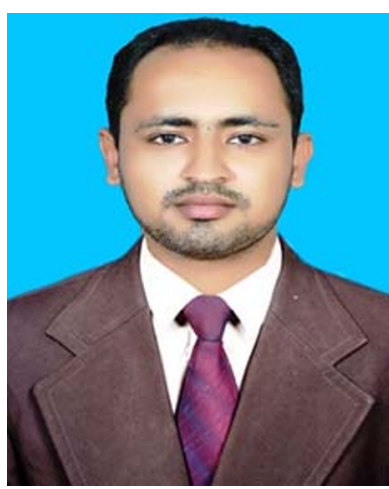

Dr. Muhammad Aqeel Ashraf is a Professor at the School of Environmental Studies, China University of Geosciences (CUG). Previously, he worked as Senior Lecturer at the Department of Geology, University of Malaya, Malaysia. He got his doctoral degree (Ph.D.) from the Department of Chemistry, University of Malaya, Malaysia, and postdoctoral from the Tokyo Institute of Technology, Japan. His current research interests are Environment and Ecosystem. $\mathrm{He}$ has published more than 300 research articles in the fields related to environment, chemistry, ecology, geology, and life sciences.

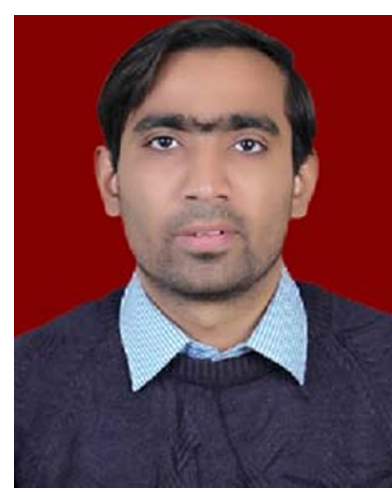

Mr. Faheem is currently pursuing a Ph.D. in Environmental Sciences and Engineering from China University of Geosciences, Wuhan. Previously, he has completed his M.Sc. (Hons.) from the School of Environmental and Biological Engineering, Nanjing University of Science and Technology, Nanjing, China. During his master and doctoral training period (2014-2020), his research was mainly focused on the valorization of different sector generated wastes to convert it into adsorbents and catalysts having a potential application for the sake of wastewater purification or restoration. Mr. Faheem has published over 15 peer-reviewed papers in high-impact journals. 